\title{
Tratamiento quirúrgico de la estenosis traqueal adquirida en pediatría
}

\author{
Surgical treatment of acquired tracheal stenosis in pediatrics
}

\author{
Dra. Giselle Cuestas ${ }^{a}$, Dra. Verónica Rodrígueza , Dr. Daniel Navacchiab , Dr. Patricio Bellia Munzón ${ }^{a}$ y \\ Dr. Gastón Bellia Munzón ${ }^{c}$
}

\begin{abstract}
RESUMEN
La estenosis traqueal adquirida es muy poco frecuente en la edad pediátrica y puede responder a múltiples causas; la estenosis posintubación y la secundaria a traqueotomía son las más comunes.

El manejo de la estenosis traqueal adquirida sigue siendo controvertido. El tratamiento endoscópico proporciona el alivio inmediato de los síntomas, pero se asocia a una alta tasa de recurrencia, y el uso de prótesis endoluminales puede incrementar la longitud de la estenosis y complicar una cirugía futura. Por el contrario, se han documentado buenos resultados a largo plazo con la resección traqueal con anastomosis primaria. Se describe nuestra experiencia con la resección traqueal con anastomosis término-terminal para el tratamiento de la estenosis traqueal posintubación en 8 pacientes.

Palabras clave: estenosis traqueal adquirida, cirugía, pediatría.
\end{abstract}

\begin{abstract}
Acquired tracheal stenosis is a very infrequent lesion in the pediatric age group and may be due to diverse causes, being post-intubation stenosis and stenosis secondary to tracheostomy the most common ones.

The management of acquired tracheal stenosis remains controversial. Endoscopic treatment provides immediate relief of symptoms but it is associated with a high recurrence rate, and the use of endoluminal prostheses can increase the length of the stricture complicating future surgery. Conversely, good long-term results have been documented with tracheal resection and primary anastomosis.

We describe our experience with tracheal resection followed by end-to-end anastomosis for the treatment of post-intubation tracheal stenosis in 8 patients.
\end{abstract}

Key words: acquired tracheal stenosis, surgery, pediatrics.

http: / / dx.doi.org/10.5546/ aap.2020.e72

a. Sección de Endoscopía Respiratoria, División de Otorrinolaringología.

b. Servicio de Anatomía Patológica.

c. Servicio de Cirugía.

Hospital General de Niños "Dr. Pedro de Elizalde",

Ciudad Autónoma de Buenos Aires, Argentina.

Correspondencia:

Dra. Giselle Cuestas: giselle_cuestas@yahoo.com.ar

Financiamiento: Ninguno.

Conflicto de intereses: Ninguno que declarar.

Recibido: 9-5-2019

Aceptado: 7-8-2019
Cómo citar: Cuestas G, Rodríguez V, Navacchia D, Bellia Munzón P, Bellia Munzón G. Tratamiento quirúrgico de la estenosis traqueal adquirida en pediatría. Arch Argent Pediatr 2020;118(1):e72-e76.

\section{INTRODUCCIÓN}

La estenosis traqueal (ET) es la disminución del calibre de la vía aérea a nivel de la tráquea. Puede ser congénita o adquirida. Esta última es rara en pediatría. En los infantes y niños pequeños, usualmente, es secundaria a la cánula de traqueotomía y, en los niños mayores o adolescentes, se asocia al uso del tubo endotraqueal de diámetro mayor del que corresponde o al empleo del tubo con balón. ${ }^{1,2}$ Otras causas, como infección (tuberculosis, histoplasmosis), quemadura, traumatismo, cirugía previa, enfermedad sistémica (enfermedad de Wegener) o idiopática, son aún más raras. ${ }^{2-4}$

Debe pensarse en esta patología en todo niño con el antecedente de intubación o instrumentación de la vía aérea que presenta estridor bifásico y dificultad respiratoria. El diagnóstico se confirma con la traqueobroncoscopía. ${ }^{2}$

El tratamiento debe ser individualizado e interdisciplinario; depende del grado de afectación clínica, del tipo de lesión, de la longitud, de la causa y de la presencia de malformaciones asociadas. ${ }^{2}$ Existen varias modalidades terapéuticas.

La resección traqueal con anastomosis término-terminal (RT-TT) es el tratamiento de elección de la ET posintubación. ${ }^{5-7}$ La longitud de la ET es el factor determinante más importante de la resecabilidad. ${ }^{8}$ Existe consenso en que las estenosis menores del $50 \%$ de la longitud traqueal se pueden tratar con resección y anastomosis primaria, mientras que, cuando la estenosis involucra más del $50 \%$ (estenosis larga), es necesaria la traqueoplastía por deslizamiento. ${ }^{9}$

\section{CASOS CLÍNICOS}

Se presentan 8 pacientes (6 masculinos) con ET adquirida tratados quirúrgicamente por la 
Sección de Endoscopía Respiratoria y el Servicio de Cirugía entre diciembre de 2010 y diciembre de 2018.

La intubación endotraqueal fue la causa de la estenosis en todos los casos, y varió el tiempo de intubación de 4 a 49 días (media: 15 días). En 7 casos, el tubo utilizado tenía balón. La indicación de la asistencia respiratoria mecánica fue traumatismo encéfalo-craneano en 3 casos, patología respiratoria en 2 casos y meningoencefalitis, quemadura y preeclampsia en 1 caso cada uno. Tres pacientes estaban traqueotomizados.

Todos los pacientes fueron evaluados con endoscopía bajo anestesia general (con instrumental rígido) para determinar la localización y la extensión de la lesión. En 7 casos, la lesión comprometía la tráquea cervical y, en 1 caso, la tráquea distal (Figura 1. A y B).

En 6 pacientes, se realizó el tratamiento endoscópico previo. La dilatación con tubos endotraqueales fue la técnica más utilizada (5 casos). En 1 paciente, se realizó la incisión de la cicatriz con electrocauterio y dilatación con balón $\mathrm{y}$, en 1 paciente, se extrajo una prótesis de silicona colocada en otra institución y, a continuación, se dilató con tubo endotraqueal (Figura 1. C y D). La intervención endoscópica permitió aliviar los síntomas y delimitar la lesión, pero no fue eficaz en la resolución de la estenosis. Ante la recurrencia de los síntomas (estridor, disnea), se decidió la corrección quirúrgica, la cual se llevó a cabo 1 o 2 meses después del procedimiento endoscópico.

La edad media al momento de la cirugía fue de 10 años (rango: de 8 meses a 17 años). El tratamiento en todos los casos consistió en la resección de los anillos traqueales comprometidos y anastomosis término-terminal en 1 tiempo quirúrgico (Figura 2).

El abordaje fue por cervicotomía en 7 casos y por esternotomía con circulación extracorpórea en 1 caso. Fueron resecados entre 2 y 8 anillos (media: 5 anillos) (Figura 3). La anastomosis libre de tensión se logró mediante la movilización traqueal distal. Un paciente requirió, además, una maniobra de descenso laríngeo. No hubo complicaciones intraoperatorias.

En el posoperatorio, se trasladó a los pacientes a la Unidad de Cuidados Intensivos; se mantuvo la intubación endotraqueal por 48 horas y se indicó la posición cefálica neutra o en flexión,

Figura 1. A y B. Imágenes endoscópicas. A. Estenosis en la tráquea proximal. B. Estenosis en la tráquea distal. C y D. Tratamientos endoscópicos. C. Prótesis de silicona. D. Dilatación con balón

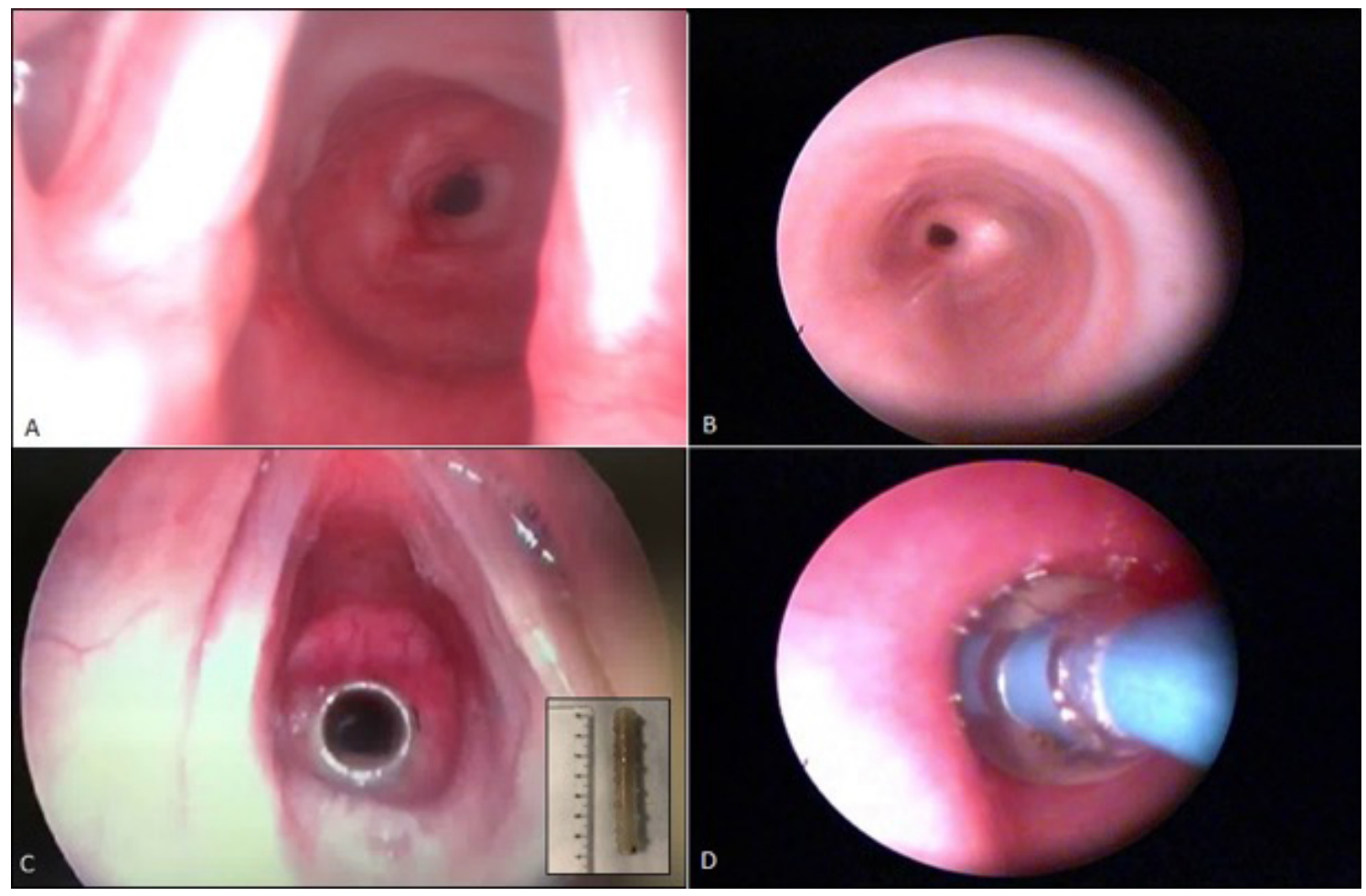


Figura 2. A y B. Imágenes endoscópicas. A. Estenosis traqueal previa a la cirugía. B. Calibre traqueal adecuado poscirugía. C y D. Imágenes intraoperatorias. C. Resección de los anillos afectados. D. Anastomosis término-terminal. Se realizan puntos separados en la cara posterior y después en la cara anterior, de medial a lateral

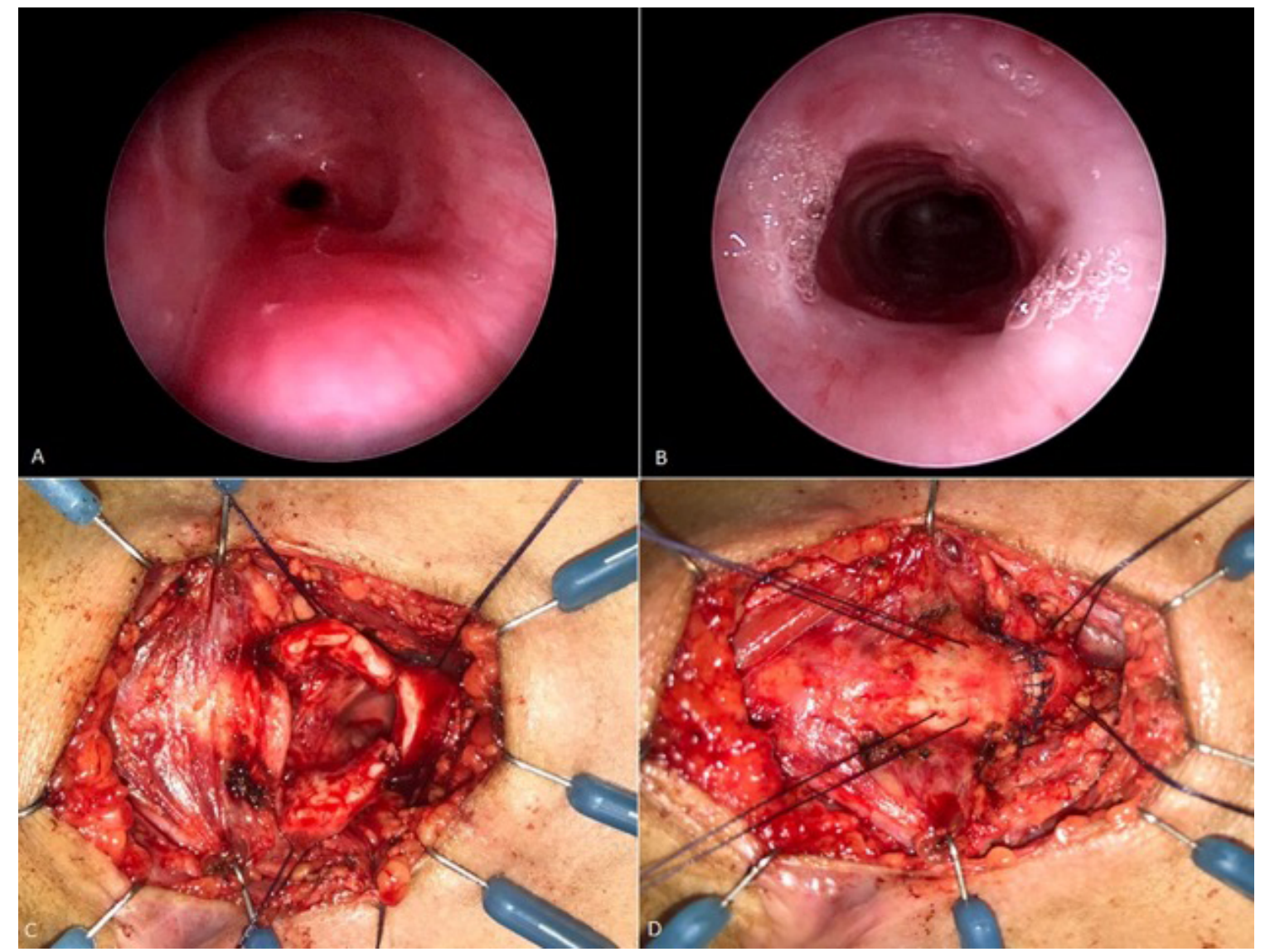

Figura 3. A. Anillos traqueales resecados. B, C y D. Histopatología. B. Se observa la tráquea con metaplasia pavimentosa epitelial, infiltrados mononucleares en el corion y un foco de metaplasia ósea subadventicial. C. Se observa el epitelio respiratorio con infiltrados mononucleares en el corion. D. Se observa un granuloma gigantocelular de tipo cuerpo extraño
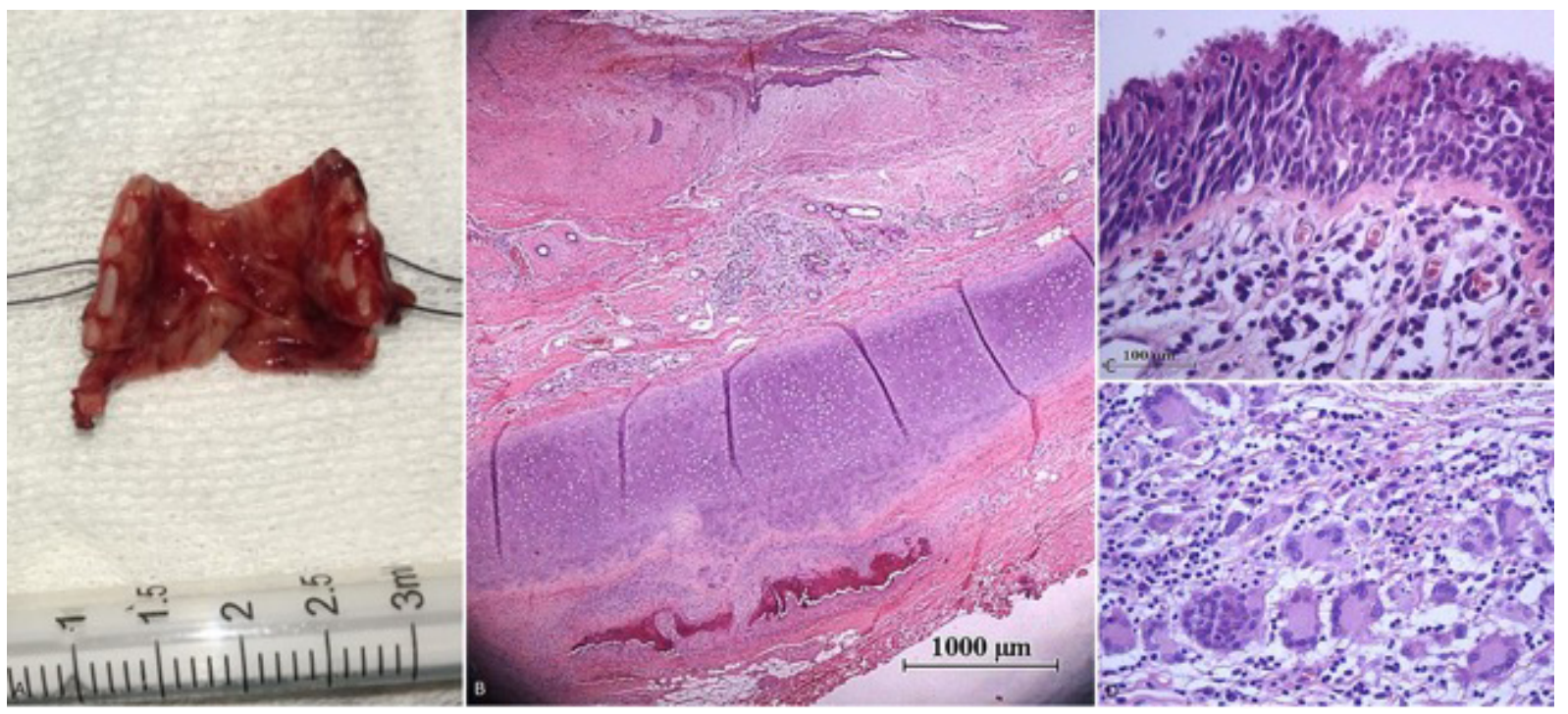
tratamiento antibiótico y antirreflujo. El tiempo de internación varió de 7 a 10 días.

Se realizaron controles endoscópicos al mes de la cirugía y a los 3, 6, 12 y 24 meses. Dos pacientes presentaron granulomas en el sitio de la sutura, que se resolvieron mediante la resección endoscópica, y un paciente presentó parálisis cordal unilateral transitoria.

El tiempo medio de seguimiento fue de 4 años (de 6 meses a 8 años). La decanulación fue posible en los 3 pacientes traqueotomizados. Todos los pacientes se encuentran asintomáticos.

\section{DISCUSIÓN}

La frecuencia de ET posintubación varía del $0,6 \%$ al $21 \% .{ }^{6}$ Es una causa poco común de estridor y dificultad respiratoria en pediatría. La lesión se produce, generalmente, en la zona que está en contacto con el balón del tubo endotraqueal por necrosis isquémica..$^{3,10} \mathrm{La}$ estenosis es típicamente circunferencial. ${ }^{2}$ Otros factores de riesgo de la ET posintubación son la intubación prolongada, la intubación traumática, el tamaño del tubo, la infección local, el reflujo gastroesofágico (RGE) y el movimiento del tubo. ${ }^{2,3,11}$

Los síntomas principales de la ET son el estridor bifásico, la disnea, la tos y/o las infecciones respiratorias recurrentes. El diagnóstico es fundamentalmente endoscópico. Permite visualizar de manera directa la lesión y determinar el grado de estrechamiento de la luz, el estado de la mucosa, la longitud y la distancia de la estenosis con respecto a las cuerdas vocales y a la carina. . $3,12^{2}$

Los estudios por imágenes (tomografía computada o resonancia magnética) proporcionan información precisa de la localización y extensión de la lesión y de las estructuras que rodean la vía aérea. ${ }^{2}$ No obstante, no siempre son adecuados, pues requieren la sedación o la intubación, lo que representa un problema en un niño disneico.

En las pruebas de función pulmonar, se observa, en la curva flujo/volumen, un aplanamiento de la curva en inspiración y en espiración, que conforma la curva cajón característica de la obstrucción fija de la gran vía aérea. $^{3}$

Dentro del diagnóstico diferencial de ET, se deben incluir otras causas de obstrucción de la vía aérea, como la patología laríngea, la traqueomalacia, la compresión traqueal vascular o por masa mediastínica y los tumores traqueales, entre otros. En ocasiones, al paciente se le puede diagnosticar erróneamente asma de control difícil o bronquitis crónica recurrente. La persistencia de los síntomas a pesar del tratamiento médico, junto con una alta sospecha clínica, debe facilitar el diagnóstico correcto. ${ }^{3}$

Generalmente, el paciente con ET se presenta con antecedente de intubación, imposibilidad de extubación, estridor posextubación o con traqueotomía y diagnóstico conocido de ET. ${ }^{12} \mathrm{El}$ manejo de la ET debe ser enfocado por un equipo multidisciplinario que incluya endoscopistas, cirujanos, otorrinolaringólogos y neumonólogos. ${ }^{12}$ La ET sintomática requiere el tratamiento endoscópico o quirúrgico. ${ }^{2,12}$

El tratamiento endoscópico (p. ej.: dilatación rígida o con balón, incisión con láser) tiene un rol limitado en el manejo de la ET debido a la alta tasa de recurrencia (del $90 \%$ en las estenosis con afectación cartilaginosa). ${ }^{6}$ Es de utilidad para aliviar los síntomas hasta el momento de la cirugía y puede ser una alternativa en los pacientes que no son candidatos a cirugía (comorbilidades), en la recurrencia poscirugía y en la estenosis simple (limitada a la mucosa, sin compromiso cartilaginoso ni malacia) en la que puede ser curativa (del $60 \%$ al $100 \%$ )., $3,5-7$

La colocación de una prótesis endoluminal (stent) debe ser considerada como una solución temporal o como último recurso en la estenosis benigna. ${ }^{13}$ La más utilizada es la de silicona y se indica en los pacientes que no responden a la dilatación endoscópica y, a su vez, no están en condiciones para la cirugía. ${ }^{3}$ Entre sus complicaciones, se incluyen la migración, la formación de granulomas, la mucostasis y el riesgo de incrementar el largo de la estenosis, lo que puede complicar una cirugía futura. ${ }^{6}$

Cuando la respuesta al tratamiento endoscópico no es adecuada o existe afectación de los cartílagos traqueales, la cirugía es el método de elección. ${ }^{4}$ La RT-TT es un procedimiento seguro cuando es realizado por un cirujano experimentado, con una tasa de éxito mayor del $95 \% .{ }^{14}$ En general, basta un solo procedimiento quirúrgico para la resolución de la ET; en la mayoría de los casos, se realiza a través de cervicotomía y en 1 tiempo quirúrgico. Resulta en una vía aérea óptima permanente y requiere una corta estancia hospitalaria. ${ }^{1,9}$

A pesar de que la resección traqueal tiene los mejores resultados a largo plazo, no es siempre posible. ${ }^{13}$ Los segmentos estenóticos cortos (menores del $30 \%$ de la longitud traqueal) representan la mejor indicación para una 
resección y anastomosis segura. Sin embargo, el límite de la resección puede incrementarse hasta el $50 \%$ de la longitud traqueal (de 8 a 10 anillos) cuando se realizan maniobras de liberación (traqueales, laríngeas y, a veces, hiliares). En las estenosis de gran longitud (mayores del $50 \%$ ), la traqueoplastía por deslizamiento ha mostrado buenos resultados y es considerada el tratamiento de elección. . $^{1,912}$

Entre las complicaciones de la RT-TT (del $14 \%$ al $39 \%$ ), se incluyen la lesión del nervio recurrente, la dehiscencia, la formación de tejido de granulación y la reestenosis.,14 Evitar la tensión de la sutura y realizar controles endoscópicos son claves para prevenir complicaciones. . $^{10,14}$

La prevención de la ET es fundamental. Evitar el tubo con balón en los menores de 8 años o su uso a baja presión ha disminuido la incidencia de estenosis posintubación. ${ }^{15} \mathrm{El}$ control del RGE también es importante. ${ }^{2,3}$

En nuestra serie de casos, la dilatación endoscópica fue de utilidad para limitar la lesión y aliviar los síntomas hasta el momento de la cirugía. La RT-TT resultó ser un procedimiento seguro y eficaz para el tratamiento de la ET adquirida en pediatría. La evaluación endoscópica minuciosa, la resección de la totalidad de la lesión (anastomosis entre tejidos sanos), la adecuada movilización traqueal y, si era necesario, de la laringe, para lograr una anastomosis libre de tensión, la disección cercana a la tráquea para evitar la lesión de los nervios recurrentes y el seguimiento endoscópico adecuado fueron los pasos fundamentales para lograr los buenos resultados.

\section{REFERENCIAS}

1. MonnierP.Tracheal resection and anastomosis. En Monnier P (ed.). Pediatric Airway Surgery. Berlín, Heidelberg: Springer-Verlag; 2011.Págs.337-47.

2. Cabezalí Barbancho D, Antón-Pacheco J, López Díaz M, Tejedor Sánchez R, et al. Estenosis traqueal adquirida: estrategia diagnóstica y terapéutica. Cir Pediatr. 2007; 20(1):19-24.

3. Barros Casas D, Fernández-Bussy S, Folch E, Flandes Aldeyturriaga J, et al. Patología obstructiva no maligna de la vía aérea central. Arch Bronconeumol. 2014; 50(8):345-54.

4. García Hernández G. Patología traqueal. Tratamiento médico y manejo clínico. An Pediatr (Barc). 2010; 72(Esp Cong 1):37-8.

5. Ulusan A, Sanli M, Isik AF, CelikIA, etal.Surgical treatment of postintubation tracheal stenosis: A retrospective 22-patient series from a single center. Asian J Surg. 2018; 41(4):356-62.

6. Özdemir C, Kocatürk CI, Sökücü SN, Sezen BC, et al. Endoscopic and surgical treatment of benign tracheal stenosis: A multidisciplinary team approach. Ann Thorac Cardiovasc Surg. 2018; 24(6):288-95.

7. Negm H, Mosleh M, Fathy H. Circumferential tracheal resection with primary anastomosis for post-intubation tracheal stenosis: study of 24 cases. Eur Arch Otorhinolaryngol. 2013; 270(10):2709-17.

8. Wain JC Jr. Postintubation tracheal stenosis. Semin Thorac Cardiovasc Surg. 2009; 21(3):284-9.

9. Boglione M, Reusmann A, Cadario M, Botto H, et al. Tratamiento quirúrgico dela estenosis traqueal en pediatría. Arch Argent Pediatr. 2010; 108(2):148-52.

10. Ahn HY, Su Cho J, Kim YD, I H. Surgical outcomes of post intubational or post tracheostomy tracheal stenosis: Report of 18 cases in single institution. Ann Thorac Cardiovasc Surg. 2015; 21(1):14-7.

11. Farzanegan R, Feizabadi M, Ghorbani F, Movassaghi M, et al. An overview of tracheal stenosis research trends and hot topics. Arch Iran Med. 2017; 20(9):598-607.

12. Delgado Pecellín I, González Valencia JP, Machuca Contreras M, Pineda Mantecón M. Clínica, diagnóstico y tratamiento de las estenosis traqueales. An Pediatr (Barc). 2009; 70(5):443-8.

13. Fernando HC, Sherwood JT, Krimsky W. Endoscopic therapies and stents for benign airway disorders: where are we, and where are we heading? Ann Thorac Surg. 2010; 89(6):S2183-7.

14. Siciliani A, Rendina EA, Ibrahim M. State of the art in tracheal surgery: a brief literature review. Multidiscip Respir Med. 2018; 13:34.

15. Monnier P. Acquired post-intubation and tracheostomyrelated stenoses. In Monnier P(ed.). Pediatric Airway Surgery. Berlín, Heidelberg: Springer-Verlag; 2011.Págs.183-98. 